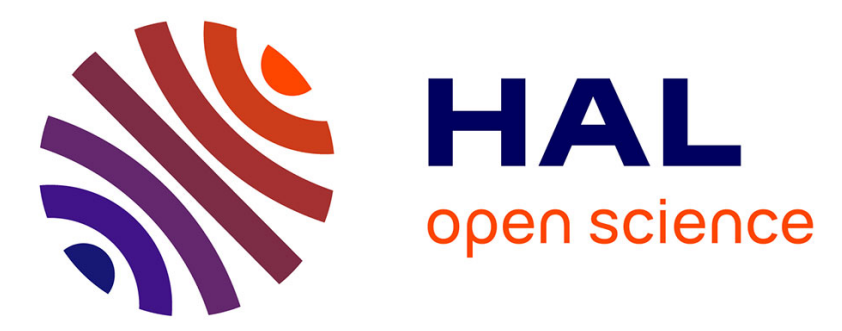

\title{
Cylindrical cyclic acoustic imaging with a Bayesian approach for cyclostationary sources reconstruction.
}

Sébastien Personne, Jérôme Antoni, Jean-Daniel Chazot

\section{To cite this version:}

Sébastien Personne, Jérôme Antoni, Jean-Daniel Chazot. Cylindrical cyclic acoustic imaging with a Bayesian approach for cyclostationary sources reconstruction.. 21st International Congress on Acoustics, Jun 2013, Montreal, Canada. pp.3546, 10.1121/1.4806426 . hal-01021432

\section{HAL Id: hal-01021432 \\ https://hal.science/hal-01021432}

Submitted on 9 Jul 2014

HAL is a multi-disciplinary open access archive for the deposit and dissemination of scientific research documents, whether they are published or not. The documents may come from teaching and research institutions in France or abroad, or from public or private research centers.
L'archive ouverte pluridisciplinaire HAL, est destinée au dépôt et à la diffusion de documents scientifiques de niveau recherche, publiés ou non, émanant des établissements d'enseignement et de recherche français ou étrangers, des laboratoires publics ou privés. 


\title{
CYLINDRICAL CYCLIC ACOUSTIC IMAGING WITH A BAYESIAN APPROACH FOR RECONSTRUCTION OF CYCLOSTATIONARY SOURCES
}

\begin{abstract}
Standard acoustic imaging techniques, such as beamforming or near-field acoustical holography, are now widely used in engineering contexts. However, large arrays of microphones are sometimes required to have a good resolution. Besides new challenges arise, particularly in the field of non-stationary sources, which need to be identified and solved.

Cyclostationary sound sources, a specific kind of non-stationary signals, are characterized by statistical properties evolving periodically in time. In practice the first-order statistical properties contain some periodic components while the second orders may be random with a periodic flow of energy. The present work tackles the acoustic imaging of cyclostationary sources with a scanning microphone, ie. without any array. Cylindrical surfaces, adapted to standard rotating machines, are considered. The difficulty of reconstructing non-stationary acoustic sources from discrete measurements is then alleviated suing their cyclostationary properties. A cyclic sound field is hence extracted that can show how sources are evolving in space and time.

Finally, a Bayesian formulation, gathering both physical and probabilistic information on the inverse problem, is used to back-propagate the sound over the radiating surface.
\end{abstract}

\section{Introduction}

\section{a. Objective}

Considering the development of a rotating machine, reduction of noise radiation is more and more a topic of concern. This requires the identification and localization of sources of noise.

A useful framework has been developed for signals coming from rotating machine (see [1]) that can be modeled as

$$
x(t)=p(t)+n(t)
$$

with $p(t)$ a deterministic part which can be correlated with a cyclic frame event and $n(t)$ a random part (for example friction, etc...). The context of concern in this paper is a product development not a failure analysis, thus the periodic part is assumed to be dominant compared to the random part. The goal is to localize the periodic sources radiating noise in space and time (what the author call "cyclic events").

In this context acoustical imaging is an efficient tool to localize sources of vibration from pressure measurement. There is a lot of methods which are unified by the author of [3] with the Bayesian formalism. For a planar geometry, the theory is strongly explained and the efficiency has been well observed [4]. As far as the authors know this process has not been built for a cylindrical shape; the following article will focus on this point. Readers can find a specific case corresponding to the Statistical Optimized Near-Field Acoustic in cylindrical coordinate in [6]. Usually, specificity of cyclostationary signals is not taken into account and this entails information loss. To overcome this problem, the Authors in [7] have developed the Near-field acoustic holography for cyclostationary sound field and the source reconstruction is presented in the frequency domain.

The Authors will use another strategy taking advantage of filtering the periodical part of the signal: the Fourier transform can be applied on this part of the signal, apply the image processing in the frequency domain and then use inverse Fourier transform to come back in cyclic time domain. This leads to reconstruction of the cyclic source field in space and cyclic time. Figure 1 illustrates the global procedure.

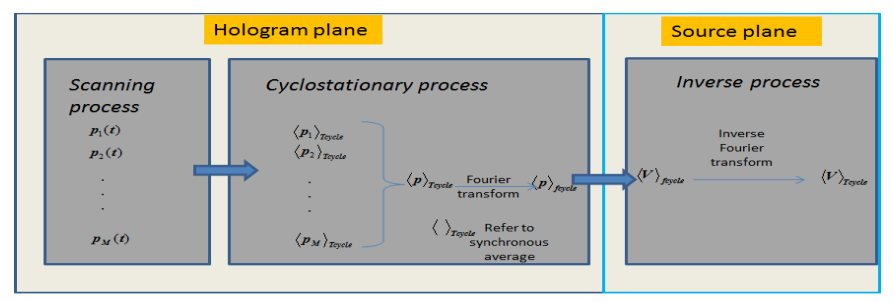


FIGURE 1: synopsis of the procedure

\section{b. Cyclostationary: definition and properties}

This paragraph is a very short introduction to Cyclostationarity and its properties. For more details on the cyclostationary analysis the reader can refer to [1], [2]. Cyclostationarity gives a theorical framework to processes whose statistical properties are periodically varying in time. By definition it embodies a class of non-stationary stochastic processes, with stationary and deterministic periodic processes as specific cases.

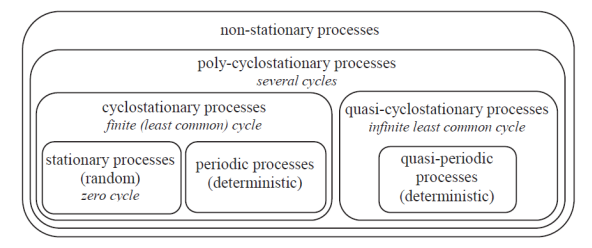

FIGURE 2: Typology of stochastic processes

When defined on the first order it means that the signal contains some periodic component then CS1 will refer to the deterministic part of the cyclostationary process; on the second order it means the signal may be random while energy is flowing periodically with time.

A formal definition can be done in terms of the expectation operator. A stochastic process, ${ }^{x(t)}$, this process is said to be cyclostationary of order $\mathrm{i}$ and of period $\mathrm{T}$ if any of its $\mathrm{i}$-th order statistics is a periodic function of time with main period $\mathrm{T}$. In this work only the first-order cylostationarity will be considered. Condensing the expression with expected value gives, $m_{x}(t) \hat{=} E\{x(t)\}$ exists and is periodic of period T, i.e.

$$
m_{x}(t)=m_{x}(t+T)
$$

In practice the only way to estimate the expected value is to perform a time synchronous averaging with the cycloergodicity assumption.

A stochastic process, $x(t)$, is said to be cycloergodic of order 1 and period $\mathrm{T}$ if its time-synchronous average is deterministic, i.e.

$$
\langle x(t)\rangle_{T} \hat{=} \lim _{K \rightarrow \infty} \frac{1}{2 K+1} \sum_{k=-K}^{K} x(t+k T)=\mu_{x}(t) .
$$

With the assumption of cycloergodicity, we have

$$
E\{x(t)\}=\langle x(t)\rangle_{T}
$$

Equation (3) means that the time synchronous average is an estimator of the expected value.

Figure below shows three realizations of a cyclostationary process (left part) and the corresponding time synchronous average (right part), reader can see that the average reveal periodic phenomena. 


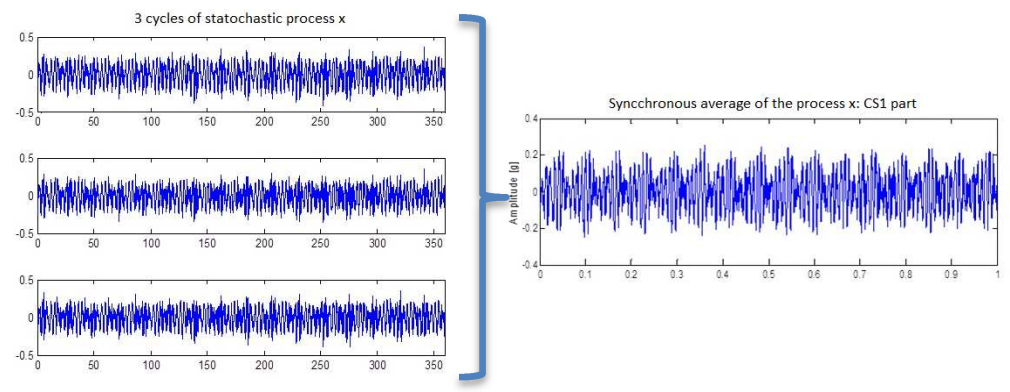

FIGURE 3: illustration of the time synchronous average and expected value construction

This points out the utility of the cyclostationary framework: since by construction the expected value is periodic, it makes possible to apply in a second step all the powerful tools developed for stationary signals such as the Fourier transform and spectral analysis.

Initially developed for communication signals, this approach seems well adapted for mechanical rotating machines. Due to the kinematics of machines, the resulting acoustic and vibration signals are periodic with respect to some angle of rotation and are therefore cyclostationary with respect to angle. For steady-state operating conditions, angle and time can be reversed and signals are cyclostationar with respect to time as well. The extraction of the CS1 part of the signal will be the first step of the procedure. Those periodic signals will be used for cyclic sound imaging.

\section{c. Bayesian approach to the inverse acoustic problem: optimal basis and regularization}

Localization of acoustic sources using discrete measurements of the acoustic field is a recurrent acoustic inverse problem. Many approaches exist, such as Beamforming, Near-field Acoustic Holography, Inverse Boundary Element Method, Helmholtz Equation Least-Squares, etc...Choosing the right technique depends on the acoustic source shape, the array geometry and the frequency range of analysis. However, most of the methods share a common process to solve the inverse problem:

1) the source field is reconstructed as linear combination of spatial basis functions,

2) the coefficients of the basis function are computed so as to match the measurement field at the microphones positions.

Let $s$ be a radiating source in the acoustic domain and let an array of $M$ microphones measure the acoustic field. The Green function $G$ between the source and the measurement array is supposed to be known analytically or numerically ( $n$ refers to the noise of measurement).

$$
p=\int_{\Gamma} s(r) G(r) d \Gamma+n
$$

The aim of the inverse problem is to reconstruct the continuous sound field $s(r)$ from the $M$ discrete measurements. The common formalism to get the reconstructed source $\hat{s}(r)$ can be expressed as:

$$
\hat{s}(r)=\sum_{k=1}^{x} c_{k} \phi_{k}(r) \text {. }
$$

where the unknowns of the problem are the basis functions $\phi_{k}$, their coefficients $c_{k}$ and the $x$ dimension of the basis.

The following will give a brief theorical background of the Bayesian approach used to solve for these unknowns -readers should refer to [3] for more details.

Bayesian inference is used to solve the problem by considering the unknown quantities as random variables that produce a random source field and by seeking the probability distribution $[s(c, \phi) \backslash p]$ conditioned to the observation of the measurements in the vector $\mathbf{p}$. This is the so-called posterior probability distribution, which may be viewed as a cost function whose maximization will lead to the optimal parameters $\hat{c}$ and $\hat{\phi}$ that best explain the measured data. 
By using the Baye's rule:

$$
[s(c, \phi) \backslash p]=\frac{[p \backslash s(c, \phi)][s(c, \phi)]}{[P]},
$$

Where $[p \backslash s(c, \phi)]$ is the likelihood function, reflecting the probability of observing $\mathbf{p}$ given the source field $s(c, \phi),[s(c, \phi)]$ is the a priori probability distribution of the source before the data are measured, and $[P]$ is the evidence.

It is important to notice that with this formulation, the inverse problem (inferring s(c, $\Phi)$ from p) has been turned in its direct problem (inferring $\mathbf{p}$ from s $(\mathrm{c}, \Phi)$ ).

From equation (4), $p \backslash s(c, \phi)$ has the same probability distribution as the measurement noise $\mathbf{n}$ (n is the vector of noise measurement for the array). According to the central limit theorem applied to the Fourier transform, the measurement noise in frequency domain is a circular complex Gaussian random variable. Introducing the covariance matrix $\operatorname{E}\left\{n n^{*}\right\}=\beta^{2} \Omega_{N}$ where $\Omega_{N}$ is define as spatially white noise with normalization $\operatorname{trace}\left\{\Omega_{N}\right\}=M$ so that the quantity $\beta^{2}$ reflects the mean energy of the noise.

The prior distribution $[s(c, \phi)]$ results from any knowledge the experimenter has on the source field before the experiment. A prior can be a spatial information about the radiating regions where the noise is more likely to originate. This information is introduced by an "aperture function " $\sigma_{S}^{2}(r)$ that takes positive or zero values on the source surface. This leads to the following structure for the spatial covariance function of the random source field:

$$
\mathrm{E}\left\{s(r) s\left(r^{\prime}\right)^{*}\right\}=\alpha^{2} \sigma_{S}^{2}(r) \delta\left(r-r^{\prime}\right)
$$

with normalization $\int_{\Gamma} \sigma_{S}^{2}(r) d S(r)=1$, so that $\alpha^{2}$ reflects the mean energy of the source field.

With the expression of the probability distributions, the inverse problem corresponds to the maximization of $[s(c, \phi) / p]$ with respect to $c, \phi$ and two hyperparameters $\alpha^{2}$ and $\beta^{2}$.

Hereafter, only the main results of the algorithm are reported. We define the singular value decomposition of the propagation operator $G$ through the aperture function and whitened by the covariance matrix of the noise $\Omega_{N}$ as follows:

$$
\sigma_{S}^{2}(r) G(r)^{*} \Omega_{N}^{-1 / 2}=\sum_{k=1}^{M} \lambda_{k} \psi_{k}(r) U_{k}^{*}
$$

where $\mathrm{G}$ is the vector containing the green functions from the source plane to each microphone of the array, $\lambda_{k}$ are non-negative scalars, $\psi_{k}(r)$ eigen-functions over the surface of the source, and $U_{k}$ eigen-vector such that:

$$
\begin{gathered}
\lambda_{1} \geq \lambda_{2} \geq \ldots \geq \lambda_{M} \geq 0, \\
\int_{\Gamma} \psi_{k}(r) \psi_{l}(r) \sigma_{S}^{-2} d S(r)=\delta_{k l} \\
U_{k}^{*} U_{l}=\delta_{k l} .
\end{gathered}
$$

It is proved in [6] that the optimal basis functions sought in (5) are the eigen-function of (8), i.e. $\phi_{k}(r) \equiv \psi_{k}(r)$; therefore

one has $x=M$ with functions that are orthogonal through the aperture function $\sigma_{S}^{2}(r)$ according to the definition in (10). The optimal coefficient $c_{k}$ sought in (5) are found as

$$
c_{k}=\frac{\lambda_{k}}{\lambda_{k}^{2}+\eta^{2}} U_{k}^{*} \Omega_{N}^{-1 / 2} P
$$


where $\eta^{2}=\frac{\beta^{2}}{\alpha^{2}}$ represents the noise-to-signal ratio and plays a crucial role for regularizing the problem.

To sum up results, the reconstructed source field is ultimately given by (13):

$$
\hat{s}(r)=\sum_{k=1}^{M} \frac{\lambda_{k}}{\lambda_{k}^{2}+\eta^{2}} \psi_{k}(r) U_{k}^{*} \Omega_{N}^{-1 / 2} P
$$

In the present work, the reconstructed source is the CS1 part of the sound field. So the vector $\mathbf{p}$ in (13) contains the CS1 part of the $M$ signals measured by the microphones of the array.

\section{Cylindrical cyclic acoustic imaging theory}

a. Scanning measurement for CS1 cyclostationary sound field construction

The shape of the acoustic source is a cylinder of radius $r_{s}$. The hologram surface will also be a cylinder of radius $r_{h}>r_{s}$.

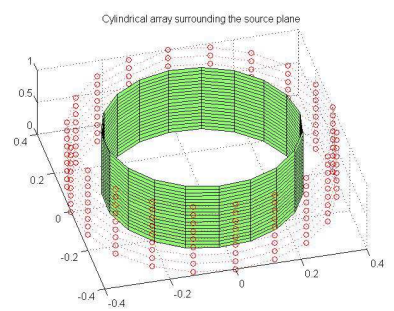

FIGURE 4: continuous source surface and discrete position for the microphones of the virtual array

The goal of the procedure is to measure and reconstruct the cyclic part of the sound field by measuring the pressure on the hologram surface by scanning a virtual array of $\mathrm{M}$ microphones, from $\mathrm{M}$ sequential measurements.

The scanned signals are not temporal synchronous, yet by construction each post processed CS1 signal is phased with a common angular event; therefore the M CS1 signals are synchronous and can be assembled in a "virtual array" of microphones.

\section{b. Bayesian approach to sound source reconstruction on a cylindrical shape}

The expression for the radiation of a cylinder is taken from [5]

$$
p(r, \theta, z)=\sum_{m=-\infty}^{m=\infty} \frac{1}{2 \pi} \int_{-\infty}^{\infty} P_{m}\left(r, k_{z}\right) e^{i m \theta} e^{i k_{z} z} d k_{z}
$$

where $P_{m}\left(r, k_{z}\right)$ is the cylindrical wave number spectrum of $p_{m}(r, \theta, z)$, the $m$-th circumferential component of the sound field. The wavenumber spectrum at radius $r$ can also be expressed in terms of the wave number spectrum of the sound field on a cylindrical source of radius $r_{s}$ :

$$
P_{m}\left(r, k_{z}\right)=\frac{H_{m}^{1}\left(k_{r} r\right)}{H_{m}^{1}\left(k_{r} r_{s}\right)} P_{m}\left(r_{s}, k_{z}\right),
$$

where $H_{m}^{1}$ is the $m$ th-order Hankel function and

$$
k_{r}=\left\{\frac{\sqrt{k^{2}-k_{z}^{2}}, \text { for }|k| \geq\left|k_{z}\right|}{\sqrt{k_{z}^{2}-k^{2}}, \text { for }|k|<\left|k_{z}\right|},\right.
$$




\section{$\underline{\omega}$}

with $\mathrm{k}=\bar{c}, \omega$ the angular frequency, $c$ the celerity and the convention for time dependence factor is $e^{-j \omega t}$

Now introducing the Euler's equation, ie,

$$
u_{r}(r)=\frac{1}{i \rho_{o} \omega} \frac{\partial p(r)}{\partial r}
$$

With $u_{r}$ the radial velocity and $\rho_{o}$ air mass density.

one obtains the modified cylindrical wave propagator,

$$
\Phi_{k_{z}, m}^{u}(r, \theta, z) \equiv \frac{-i k_{r}}{\rho_{0} \omega} \frac{H_{m}^{1}{ }^{\prime}\left(k_{r} r\right)}{H_{m}^{1}\left(k_{r} r_{s}\right)} e^{i m \theta} e^{i k_{z} z}
$$

and finally the propagator,

$$
G=\frac{1}{2 \pi} \sum_{-\infty}^{\infty} \int_{-\infty}^{\infty} \Phi d k_{z}
$$

In the computer implementation those infinite summation have to be sampled and truncated.

Note that in theory this propagator is calculated for an infinite cylinder and is not valid for a finite cylinder. However, the Baye's formalism allows introduction of a prior in the form of finite support-set aperture function along the $\mathrm{z}$ axis that forces a finite cylinder height.

The narrower the aperture function and the more localized the prior; how the optimal spatial basis changes accordingly is shown in the following figure.

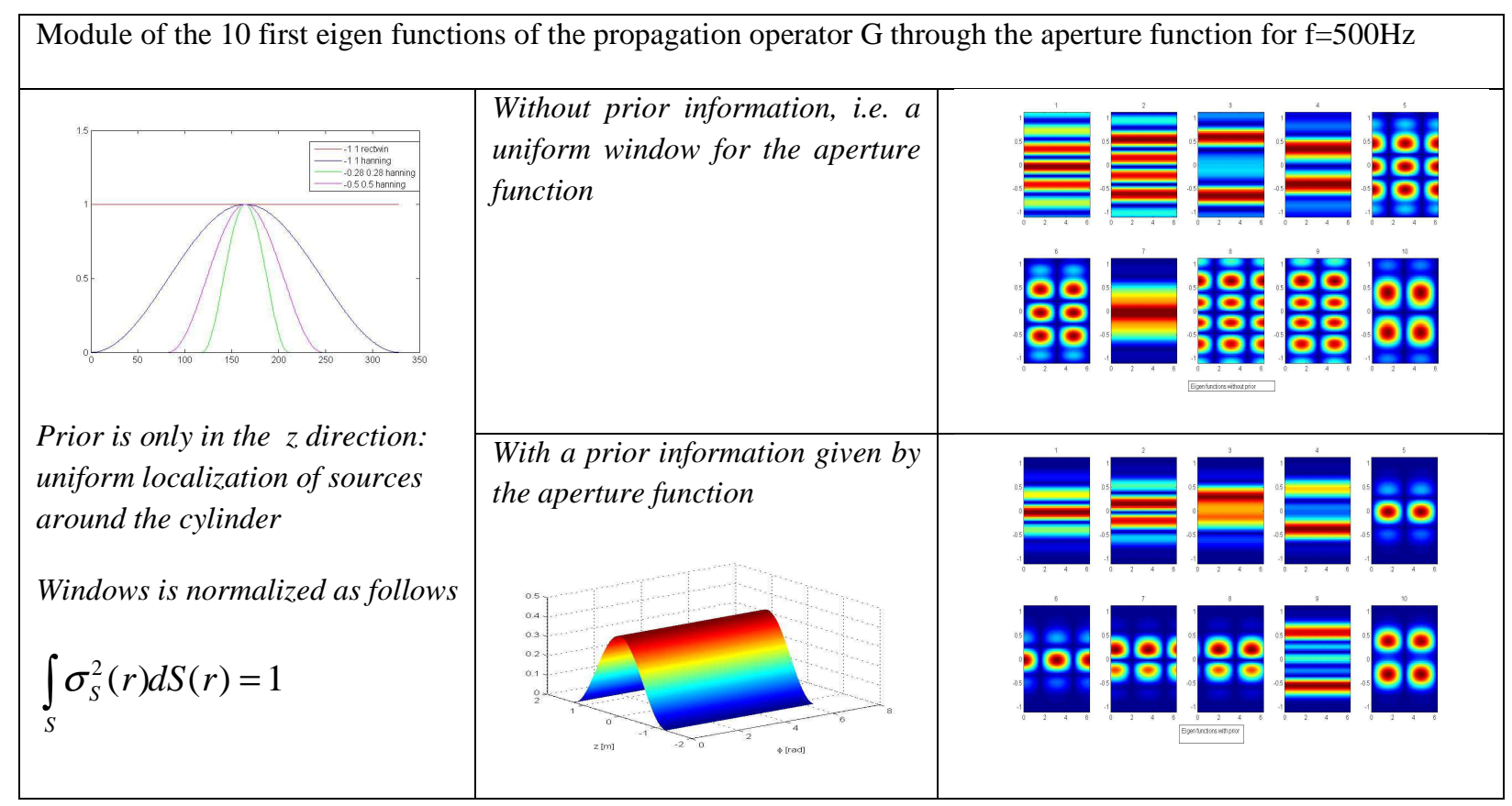




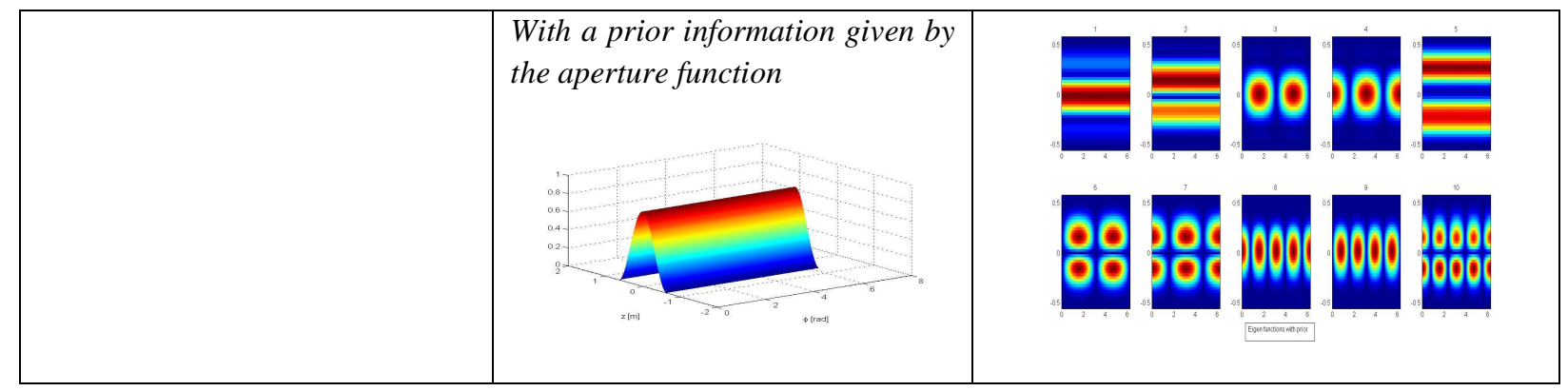

FIGURE 5: Evolution of the basis function for source reconstruction with prior on the source location along $\mathrm{Z}$ axis.

Figure 5 shows that the optimal basis strongly depends on the choice of the aperture function. Man can notice that the spatial basis corresponding to a uniform aperture function (line1 in figure 5) is the specific case use for SONAH in cylindrical coordinates [6].

\section{Numerical simulation}

\section{a. Source signal simulation}

The simulation tries to represent the behavior of a rotating machine; its housing has a cylindrical shape and is the rotating part of the machine. The rotation is due to kinematics events which cause internal forces. Each cyclic event produces a source with its localization and from an external point of view this corresponds to moving sources within the cycle.

Consider a cylinder in motion with a constant rotation speed. Two acoustic sources rotate relatively to this cylinder. For the simulation the CS1 sound field is considered only; accordingly the source variation is made periodic with one rotation but frequency change within this period. The speed is $120 \mathrm{rpm}$ and Fs $=2 \mathrm{kHz}$ (sampling frequency);

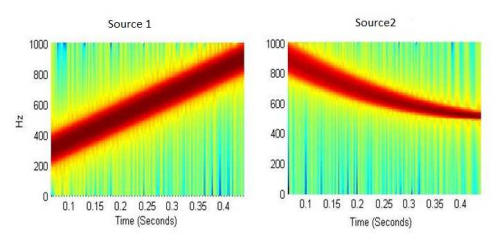

FIGURE 6: Evolution of sources with respect to time and associated instantaneous frequency. Signals are shown over 1 cycle, $\mathrm{T}_{\text {cycle }}=0,5 \mathrm{~s}$.

From the fix point of view of a sensor on the array, man see two acoustics sources following a circular trajectory as shows the figure 7.

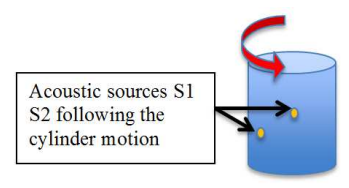

FIGURE 7: geometry used in simulations

\section{b. Source reconstruction by Bayesian approach}

The Eulerian point of view is used to obtain a clearer idea of the source localization at one particular instant. Gathering successive pictures give a movie of the source motions. Figure 8 presents two pictures taken for sample 100 and sample 900. 


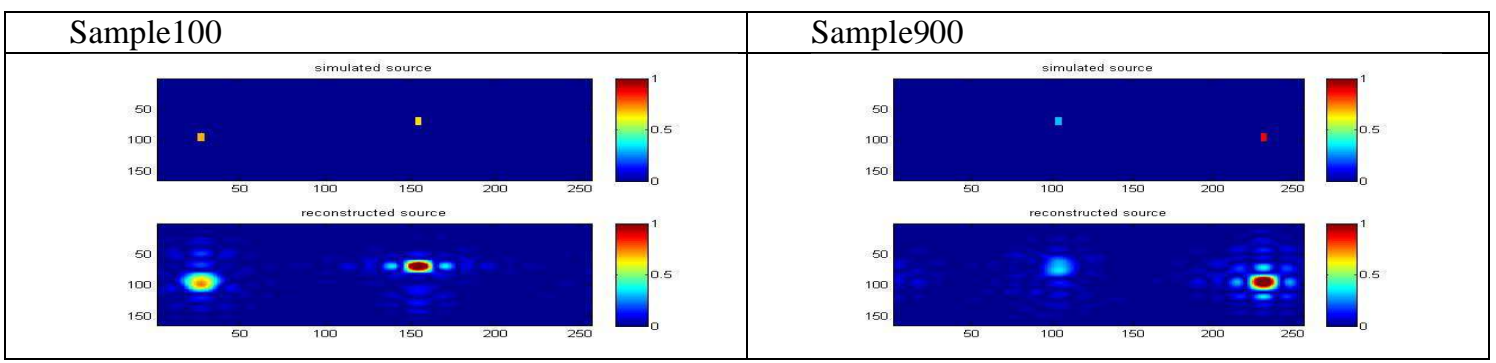

FIGURE 8: comparison of simulated source field and the reconstructed source field for two specific time in the cycle

Taken the Lagrangian point of view we see the accuracy of the time varying reconstruction of the process. Figure 9 shows the evolution of the particle velocity as if the sensors stuck to each source during motion.

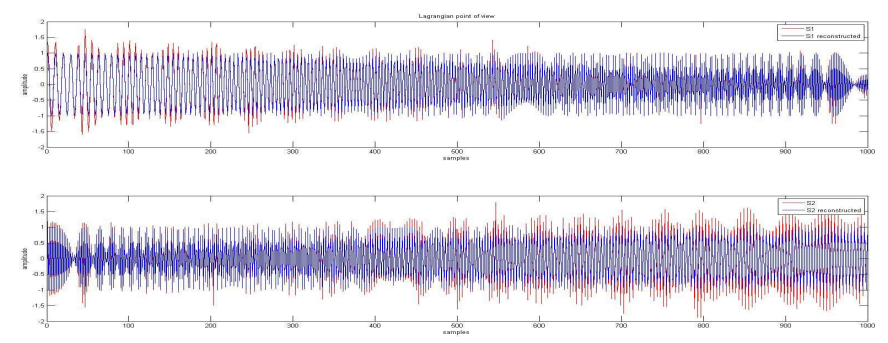

FIGURE 9: reconstruction of each source in time

\section{Conclusion}

Present work gives a framework for the analysis of a rotating machine with housing as rotating part. This will take advantage of cyclostationary properties of signal generated by rotating machine to localize the acoustical sources in space and time.

A simulation demonstrates the ability to reconstruct the sound field over a cylindrical surface from a periodic field measured in cylindrical surface surrounding the source.

This simulation has to be completed with a test on real source to evaluate the global process: from scanning measurement to sound field reconstruction. Particularly the influence of the phase error due to the angular resampling process, see [8]. Reader can see in [9] that, measurement done by step and synchronized by a precise angular event enable to find an accurate source location in a gear power transmission. Author's work will be to follow the same way with a "Cylindrical robot" for a cylindrical source.

\section{REFERENCES}

1. J.Antoni, "Cyclostationary modeling of rotating machine vibration signals", Mechanical System and Signal Processing 18, 1285-1314(2004).

2. J.Antoni, "Cyclic spectral analysis in practice", Mechanical System and Signal Processing 21, 597-630(2007).

3. J.Antoni, "Focalisation Bayésienne: une approche unifiée du probleme inverse en acoustique » (in French), 10 éme Congrés Français d'Acoustique (2010)

4. J.Antoni, “A Bayesian approach to sound source reconstruction: Optimal basis, regularization and focusing" J. Acoust. Soc. Am. 131 2873-2890 (2012)

5. E. Williams, « Fourier Acoustics sound radiation and nearfield acoustical holography », chapters $4 \& 5$ (1999)

6. Y. Cho,"Source visualization by using statistically optimized near-field acoustical holography in cylindrical coordinates", Acoustical Society of America, 2355-2364 (2005)

7. Q.Wan,"Near field acoustic holography(NAH) for cyclostationary sound field and its application”, J of sound and vibration 290, 956-967 (2006)

8. F.Bonnardot,"comparaison entre les analyses angulaire et temporelle des signaux vibratoires de machines tournantes, etude du concept de cyclostationnarité floue"(in French), $\mathrm{PhD}$ dissertation institute national polytechnique de Grenoble (2004)

9. M.Boucaud, »Improved acoustical sources location in gear power transmissions », Vibration, Shocks\&Noise (2012) 